\title{
Y a-t-il une durée optimale d'hospitalisation en post-partum ?
}

\section{Considerations about the Duration of the Postnatal Stay at the Hospital}

\author{
N. Moreau $\cdot$ S. Alexander \\ (C) Lavoisier SAS 2015
}

Le nouveau numéro de la revue a comme thème la période postnatale.

Ce n'est pas un thème nouveau. Dans la religion chrétienne et dans la religion juive, une période de 42 jours était dévolue à l'accouchée, qui ne sortait pas de chez elle. Chez les chrétiens, cette période se terminait par la fête des « relevailles », qui correspond à la présentation au temple de Jésus, et qui est fêtée six semaines après Noël, soit le 2 février (la chandeleur). C'est dire que, dans notre culture, a été reconnue et observée une sorte de « parenthèse temporelle », avant que la mère et son enfant ne rentrent dans le monde de tous les jours.

Après la Seconde Guerre mondiale, un changement important s'est opéré en Europe et la vaste majorité des femmes a cessé d'accoucher à la maison pour le faire à l'hôpital. Dans un premier temps, elles restaient à l'hôpital environ une semaine. La justification de cette durée relevait de trois domaines. Premièrement, les soignants pouvaient surveiller la santé physique de la mère : suture abdominale ou périnéale, hémorragie secondaire, infection; et de l'enfant : ictère, détresse respiratoire, infection, mauvaise prise pondérale. Deuxièmement, ils étaient vigilants au sujet des difficultés psychologiques ou émotionnelles : baby blues, troubles de l'attachement. Finalement, les soignants jouaient un rôle de promotion de la santé dans les nombreux accompagnements nécessaires en période périnatale : allaitement, identification de la nature des pleurs, apprentissage des gestes de puériculture, etc.

Il faut savoir que cette durée de cinq à six jours en postpartum était spécifique à certains pays d'Europe : France, Belgique, pays latins et pays de 1'Est [1]. Dès les années 1960, les séjours courts, certainement de moins de 72 heures,

\footnotetext{
N. Moreau $(\bowtie) \cdot S$. Alexander

École de santé publique - ULB, route de Lennik 808 CP 596, 1070 Bruxelles, Belgique

e-mail : nathalie.moreau@ulb.ac.be

S. Alexander

Office de la naissance et de l'enfance
}

sont devenus la règle aux États-Unis, dans les pays nordiques ainsi qu'au Royaume-Uni.

Bien que les objectifs du suivi en post-partum ne soient pas remis en cause, une réflexion est menée actuellement pour savoir s'ils ne peuvent être réalisés aussi bien, voire mieux, au domicile dans le cadre d'un accouchement sans complication.

Aujourd'hui, la question est de savoir s'il y a une base d'évidence suffisante concernant la durée « optimale » de séjour en post-partum. Une Revue Systématique Cochrane (RSC) ne montre aucun bénéfice au séjour long [2]. Par ailleurs, une analyse économique a été réalisée à Genève dans le cadre d'un des essais randomisés inclus dans cette RSC [3]. Cette étude a mis en évidence qu'un séjour plus court entraînait une réduction des coûts hospitaliers mais en contrepartie les coûts en santé communautaire étaient augmentés pour la coordination et la réalisation des soins postnatals à domicile.

Les défis pour les pays qui passent au séjour court sont sans doute de trois ordres : (i) comment organiser le postpartum à domicile ; (ii) comment identifier les cas pour lesquels un séjour hospitalier plus long est nécessaire (grande prématurité, par exemple) ou judicieux (vulnérabilité sociale, par exemple) ; et (iii) comment suivre et analyser l'efficacité du système, en particulier, comment identifier et remédier aux failles du système (patientes qui après la sortie de la maternité n'utilisent pas ou mal les filières de soins offertes dans la communauté).

Ce numéro de la revue contient six articles, qui répondent en tout ou en partie à ces trois questions.

L'article de Leroux illustre l'efficacité du système mis en place en Suède. Il est incontournable que les systèmes de type beveridgien avec une forte composante de santé communautaire sont particulièrement adaptés au post-partum à domicile. Retenons aussi de cet article un ratio de naissance par sage-femme de 14 au lieu des 41 en France, ce qui nous rappelle que le raccourcissement du séjour hospitalier ne doit pas avoir comme objectif de « faire des économies » mais bien de redistribuer les ressources de la manière la plus efficace et la plus satisfaisante pour les usagers et les soignants. 
Une autre composante importante relevée dans cet article est l'informatisation globale du système qui favorise la continuité des soins et leur évaluation.

Trois autres articles ont trait à la situation française. Royer rappelle que l'organisation des soins s'élabore dans le cadre de l'approche culturelle de la santé adoptée par la société. L'auteure décrit avec justesse une modalité organisationnelle fondée sur la sage-femme libérale; système qui fonctionne aussi aux Pays-Bas et en Belgique. Elle souligne le caractère essentiel de la communication opérationnelle et d'un juste salaire. Sikias et Foix l'Hélias dressent un inventaire des enjeux pédiatriques des sorties précoces. Les auteurs rappellent la nécessité de l'adhésion des parents, d'un suivi à domicile de qualité et d'un accès au système de santé optimal. L'article d'Evrard a trait aux demandes et vécus des utilisateurs. Il est globalement en accord avec d'autres enquêtes, notamment au Royaume-Uni où les femmes sont très satisfaites du suivi de la grossesse et de l'accouchement mais pas des suites de couche [4]. Au travers des témoignages, Evrard met en lien le désarroi des femmes à la perspective du retour à domicile avec la parité, la manière dont les femmes ont vécu leur accouchement et la qualité ou l'adéquation de l'accompagnement prodigué à la maternité. Par ailleurs, le témoignage de certaines mamans illustre les réticences des pédiatres à l'encontre des sorties précoces alors que celles-ci avaient été anticipées et organisées avec une sage-femme libérale. Les auteures de cet éditorial font l'hypothèse qu'une des difficultés rencontrées dans la transition des soins de l'hôpital vers la maison est liée à l'inquiétude des pédiatres, en partie possiblement parce qu'ils ont moins l'occasion de collaborer avec les sages-femmes libérales et ne perçoivent peut-être pas leurs compétences pour identifier des symptômes précoces de pathologie pédiatrique.

L'article de Gonnaud et al. porte sur l'état de stress posttraumatique (ESPT) en périnatalité et ses multiples conséquences possibles. Les auteurs présentent une étude de cas visant à donner des repères pour le diagnostic et la prise en charge de ce syndrome durant la grossesse, l'accouchement et le suivi en post-partum. Enfin, Rigouzzo s'intéresse aux douleurs aiguës en post-partum. L'auteure rappelle que ces douleurs peuvent survenir chez les femmes ayant eu une césarienne mais également chez celles qui ont accouché par voie basse. Un traitement analgésique adéquat facilite le rétablissement de la femme, et donc la mise en place de l'allaitement et du lien mère-enfant, et prévient la chronicisation de ces douleurs.

Ces deux derniers articles illustrent, si besoin était, que tous les accouchements ne sont pas simples. Certaines femmes rencontreront des difficultés dont la prise en charge ne pourra s'inscrire que dans un modèle individualisé de suivi en post-partum, quelle que soit la durée du séjour.

À la lecture de ces différents articles, il paraît raisonnable de considérer qu'en l'absence de risque, un séjour plus court à la maternité serait approprié pour autant qu'il soit désiré par la femme, anticipé en étroite collaboration avec la femme et les professionnels de la santé, et qu'un système performant et individualisé d'accompagnement à domicile soit mis en place. Il paraît dès lors peu vraisemblable de penser le retour précoce en termes d'économies.

\section{Références}

1. Health at a glance OECD http://www.oecd.org/health/health-at-aglance-europe-23056088.htm. Dernier accès 23/12/2014

2. Brown S, Small R, Argus B, et al (2002) Early postnatal discharge from hospital for healthy mothers and term infants. Cochrane Database Syst Rev (3):CD002958

3. Petrou S, Boulvain M, Simon J, et al (2004) Home-based care after a shortened hospital stay versus hospital-based care postpartum: an economic evaluation. BJOG 111:800-6

4. NPEU (2014) Safely delivered - a national survey of women's experience of maternity care 2014. https://www.npeu.ox.ac.uk/ downloads/files/reports/Safely\%20delivered\%20NMS\%202014. pdf 